\section{THE GLANDULAR FEVER OF CHILDHOOD.}

\author{
BY G. SCOTT JACKSON, M.D. GLASG.
}

THE following case appears to me to be fairly typical of this disease as described by Dr. Dawson Williams, ${ }^{1}$ and yet it presents one or two points of peculiar interest. First as to the "point of entry" of the infection. In this district at present we are passing through a well-marked epidemic of "milk infection," the sufferers presenting evident symptoms of systemic poisoning, with purging, vomiting, and high temperature, which are only relieved by the complete withholding of all milk. In several instances there are also present one or two swollen glands in the neck which [5 presume are caused by absorption of poison from the intestine. The diarrhoea, however, in these cases carries off the poison already present and the change of diet prevents any fresh introduction. Where such is not the case and constipation is present instead of diarrhoea the absorption is more complete and glandular fuver, as in the instance $I$ am about to relate, results. This child's symptoms were so very similar to those of others round about him suffering from the epidemic, with the exception that (1) he was constipated instead of purged, and (2) the swelling of the glands was more complete, that I cannot help thinking that there is some connexion between the two disorders. That the infection is derived from the intestine rather than from whe pharyns is the view held by many responsible authorities. Gecondly, as to the termination of the disorder Dr. Dawson Williams observed that this is " marked by the passage of thin greenish fæces mixed with mucus" and Dr. Charles W. Chapman ${ }^{2}$ noticed epistaxis in four out of five cases. In my case convalescence was begun by a sudden attack of lamaturia and by the voiding of two or three offensive stools. The temperature came down at once and improvement was fairly rapid.

The patient was a bealthy child, aged three years, with good family history. He went to bed apparently well on Sept. 6th, but he awoke soon after midnight screaming and seemed to be quite delirious. He vomited once or twice and was very feverish. He was a little better on the morning of the Th, but was hot and restless all day. After another bad night I was sent for on the 8th to find the child with a temperature of $102.8^{\circ} \mathrm{F}$. and a certain amount of tenderness across the bowels, which had not been moved for two days. There was no sore throat and no marked redness of the pharynx. The next day the temperature was $103^{\circ}$ and there was a tender swollen gland along the anterior border of the left sternomastoid. The bowels had been opened after a dose of castor oil, but the tongue was very coated and the child refused all food. On the 11th the whole chain of glands on the left side were enlarged and tender. The temperature was still up to $102 \cdot 6^{\circ}$ and the patient was much distressed. The right side now began to be affected and on the morning of the 19th the head seemed to be fixed by a row of hard glands on each side, the temperature reaching $103.5^{\circ}$. In the evening of the same day I was summoned to find that the patient had been seized with an attack of hæmaturia. The temperature, however, was lower $\left(102^{\circ}\right)$ and the child seemed easier. He had a fairly good night and the next morning, after the passage of two or three watery stools, the temperatare fell to normal. Though the urine contained a trace of albumin till the 20th and the swelling of the glands did not entirely subside for some little time, the child never afterwards retrogressed, but made a complete, if somewhat tardy, recovery.

Alnwick.

\section{A CASE OF ACUTE INTUSSUSCEPTION.}

\section{By Fredericas BrooKe, M.R.C.S. Exg.}

$\mathrm{C}_{A S B S}$ of acute intussusception are so grave in character that notes of those in which treatment has been attended with a successful issue will probably be of interest, especially eince, as appeared in a recent article by Dr. F. Holme
Wiggin in the columns of THE LANCET, ${ }^{1}$ opinions differ as to the best method of treating them.

On Aug. 29th last I was called to see a female child, eleven months old, who, the mother informed me, had suffered from constipation and until that morning bad not had a motion since Aug. 27th. The motion which had just been passed was lumpy in character and was slightly streaked with blood; it was not saved for my inspection. The child was well nourished but pale, was rather sunken about the eyes, and had a somewhat anxious expression. She had occasional paroxysms of pain, but in the intervals she was bright, took notice of her surroundings, and raised berself up in her cot. There was no vomiting. The pulse was not quick, but full and regular, and the skin was dry. The appetite had fallen off. Two hours before my arrival the mother bad administered a teaspoonful of castor oil. The abdomen was moderately distended and not particularly sensitive to pressure. The resonance of the colon was well marked. A provisional diagnosis of constipation associated with flatulence was made and laxatives and a carminative mixture were prescribed. At my visit about 11 A.M. on Aug. 30th the eyes were sunken and the face pale and anxious-looking. The child was in great pain, there were violent straining and tenesmus, and the buttocks were covered with blood-stained mucus which was seen to be exuding from the anus; there was frequent vomiting. On passing my forefinger into the rectum I found it almost entirely occupied by a sausage-shaped tumour round which the finger could be swept and presenting a central orifice. Pressure, however slight, on the abdomen gave rise to great pain. To effect reduction I made use of enemata of warm water combined with inversion of the patient. At the first attempt practically none of the water was retained, but as it was forcibly injected a subsequent examination showed that the tumour had receded very slightly. After a short interval another pint of water was injected slowly but forcibly, and since a certain quantity of this was retained $I$ now bad the patient inverted. Per rectum the intussusception could now only barely be reached with the tip of the forefinger. Once more the treatment was repeated, the rectum being encouraged to retain as much of the flaid as possible and inversion practised as before. A further examination failed to show any signs of the presence of the intussusception. The hips were now elevated and the child ordered to be kept perfectly quiet. On calling two hours afterwards I was informed that the patient had rested quietly, had not been sick, and was very willing to take nourishment, which was only allowed in small quantities at a time. Drop doses of tincture of opium were ordered every four hours to quiet peristalsis and the patient was allowed to suck ice. On my visit at 11 A.M. on Aug. 31st I found that the child had slept for two hours at a time and that she had pain only occasionally; the abdomen was sensitive to pressure and was moderately distended. She romited once in the early morning and once again at 6 A.M. The patient seemed brighter and the eyes were less sunken. During the day she passed flatus several times. The resonance of the colon was well marked. At 8 P.M. I was informed that there had been no further vomiting and that the child had "talked" for the first time since her attack and shown an inclination to play. Nourishment was taken readily. There was no discharge from the bowels. On Sept. 1st the child passed an almost healthy motion in the morning, the only departure from normal being the admixture of a perceptible quantity of mucus. She was langhing and playing in the mother's arms when I made my visit. The further progress of the case presented no points of interest and there has been no return of the symptoms.

West Bridgford, Notts.

I Infantile Intussusception: A Study of One Hundred and Three Cases treated either by Fnemata or Laparotom and a Report of Two Hitherto Unreported Cases, THE LaNCET, Aug. 28th, 1897, p. 025.

SLAUghter-houses IN BATH.-At a meeting of the Bath Sanitary Committee, held on Oct. 4th, the subcommittee recommended that if a person or a private company would submit plans for the erection of slaughter-houses on an approved site they should be favourably entertained. This recommendation was adopted, and a resolution was passed urging the Corporate Property Committee, who own the slaughter-houses in Parson's Yard, to treat for the surrender of the lease in order to close them. 